\title{
Heteroresistant vancomycin-intermediate Staphylococcus aureus (hVISA) in prosthetic joint infections: a case series and review
}

This article was published in the following Dove Press journal:

Orthopedic Research and Reviews

10 May 2012

Number of times this article has been viewed

Brahman Sivakumar'

Alex Chaudhuri²

Praveen Vijaysegaran ${ }^{3}$

'Department of Orthopedic Surgery, Royal Brisbane and Women's Hospital, Herston, ${ }^{2}$ Department of Infectious Diseases, The Prince Charles Hospital, Chermside, ${ }^{3}$ Department of Orthopedic Surgery, The Prince Charles Hospital, Chermside, Australia

Correspondence: Brahman Sivakumar Department of Orthopedic Surgery, The Royal Brisbane and Women's Hospital, Bowen Bridge Rd,

Herston, QLD, Australia

Tel $+6 \mid 736368$ III

Mobile +6I 423770886

Email brahman.sivakumar@gmail.com
Abstract: Prosthetic joint infections are notoriously difficult to manage and often result in prolonged antibiotic therapy, which has been identified as contributing to bacterial resistance. Heteroresistant vancomycin-intermediate Staphylococcus aureus is a substrain of methicillinresistant Staphylococcus aureus associated with a higher affinity for bone and joint infections. Here, we present three cases of heteroresistant vancomycin-intermediate $S$. aureus prosthetic joint infection at our institution, review the literature, and provide recommendations for the management of such infections in the future.

Keywords: vancomycin, antibiotics, resistance, orthopedics

\section{Introduction}

Prosthetic joint infections (PJIs) are notoriously complex to manage, due to the presence of prosthetic material, biofilms, and the deep nature of the infection, and they often result in prolonged durations of antibiotic therapy. Approximately a quarter of all PJIs are attributed to Staphylococcus aureus, with methicillin-resistant Staphylococcus aureus (MRSA) implicated in almost half of these. ${ }^{1,2}$ Predictably, this has led to an increased reliance on glycopeptides. This reliance, combined with the aforementioned inherent difficulties in management, may be responsible for the emergence of strains of $S$. aureus that demonstrate variable and reduced susceptibility to glycopeptides. ${ }^{3}$ One such subgroup of S. aureus is heteroresistant vancomycin-intermediate Staphylococcus aureus (hVISA), which is defined by the presence of subpopulations of MRSA (typically at a rate of one organism per $10^{5}-10^{6}$ organisms) with intermediate vancomycin resistance (minimum inhibitory concentration, $8-16 \mathrm{mg} / \mathrm{mL}$ ). ${ }^{2,3}$ In comparison with vancomycin-susceptible Staphylococcus aureus (VSSA), hVISA is associated with a higher proportion of bone, joint, and prosthesis infections, as well as more frequent treatment failure. ${ }^{4}$

Here, three cases of hVISA PJI at our institution are described, all resulting in unsatisfactory outcomes as this tenacious pathogen was unable to be eradicated. Interestingly, all three isolates were found to have reduced susceptibility to daptomycin, possibly rendering it redundant as an alternative treatment modality for hVISA. Then the literature is reviewed and recommendations for the management of such infections in the future are provided.

\section{Case series Case I}

An 87-year-old man underwent an elective left total-knee arthroplasty for osteoarthritis. His comorbidities included paroxysmal atrial fibrillation, hypertension, aortic sclerosis, 
asthma, gout, and a 20-pack per year smoking history. He received standard antibiotic prophylaxis with 24 hours of intravenous cefazolin. Routine nasal and groin swabs, collected perioperatively, showed no evidence of MRSA colonization.

He was discharged uneventfully and progressed well, until a fall 5 months later resulted in a left patella fracture. This was stabilized with open reduction and internal fixation. Standard antibiotic prophylaxis was again employed. Routine nasal, groin, and surgical wound swabs were taken on postoperative day 5 for infection control surveillance. The surveillance swabs grew MRSA that was sensitive to vancomycin on routine susceptibility testing (using the Vitek ${ }^{\circledR} 2$ [AST-P612; bioMérieux Australia Pty Ltd, Baulkham Hills, Australia] system). He went on to recover uneventfully and was discharged 7 days after the operation.

Unfortunately, over the following days, his wound began discharging, prompting readmission to hospital on day 10 . Suspecting deep infection, the patient was returned to the theater, where pus was found extending into the joint. An extensive debridement and a polyethylene liner exchange were performed. Intraoperative tissue samples revealed MRSA and, in the context of a deep-seated infection, screening for hVISA was performed using high inoculum Etest ${ }^{\circledR}$ (AB Biodisk, Solna, Sweden), which was found to be positive. A population analysis profile confirmed hVISA (Table 1). This was unexpected, as the patient had only received short courses of perioperative prophylaxis with cefazolin prior to this.

Postoperatively, vancomycin was commenced for 6 weeks, accompanied by pristinamycin, which was continued for the next 13 weeks. Despite ongoing therapy with vancomycin and pristinamycin, wound discharge persisted and subsequent dehiscence led to a decision to revise the joint. The first stage of this was done with component removal and the insertion of a cement spacer containing vancomycin and tobramycin. Intraoperative swabs grew Klebsiella pneumoniae and Citrobacter koseri (both sensitive to cefazolin and ciprofloxacin), but no Staphylococcal species. This led to the impression that the treatment failure was due to a concomitant Gram-negative infection. The patient received a 7-week course of ciprofloxacin, with pristinamycin continued, for the previously documented infection with hVISA. An aspiration of the joint performed 3 weeks later for joint swelling, while the patient was on ciprofloxacin and pristinamycin, revealed no microbial growth.

An assessment made 3 months after spacer insertion revealed a poorly healing wound with copious amounts of discharge. He was taken back to the theater for joint washout, tissue sampling, and spacer removal. A large infected hematoma and a significant amount of necrotic tissue were visualized during the procedure. Intraoperative tissue samples again grew hVISA. With the repeated failure of surgical and antibiotic therapies, a salvage procedure was considered as a last resort. Three days after his joint washout, a left aboveknee amputation was performed. A 3-week course of oral linezolid was completed after his amputation. Although the outcome was the loss of a limb, fortunately there were no further issues with infection at his last review, 5 months postamputation.

\section{Case 2}

A 73-year-old woman underwent an elective one-stage revision for a chronically painful hip replacement. Operative samples were sterile while off antibiotics, confirming a noninfective process. Unfortunately, MRSA wound colonization was noted on routine postoperative surveillance. This was the first time MRSA was detected in this patient. Persisting pain and new discharge from the surgical site led to a further twostage revision 3 months later. MRSA PJI was confirmed in positive cultures from the explanted prosthesis. Vancomycin was administered for 6 weeks between the two stages of the procedure, with normalization of C-reactive protein.

Unfortunately, 3 months later, a clinical relapse of the infection was noted. The patient returned to the theater for washout and debridement, with intraoperative cultures again revealing MRSA infection. A repeat two-stage revision was performed and the patient received 6 weeks of vancomycin

Table I Vancomycin and daptomycin susceptibility patterns for isolates from three cases

\begin{tabular}{|c|c|c|c|c|c|}
\hline \multirow[t]{3}{*}{ Isolates } & \multicolumn{4}{|c|}{ Minimum inhibitory concentration (mg/L) } & \multirow[t]{3}{*}{$\overline{\text { PAP (AUC ratio) }}$} \\
\hline & \multicolumn{2}{|c|}{ Vancomycin } & \multirow{2}{*}{$\frac{\text { Teicoplanin }}{\text { Macro Etest }^{\circledR}}$} & \multirow{2}{*}{$\frac{\text { Daptomycin }}{\text { Vitek }^{\circledR} \mathbf{2}}$} & \\
\hline & Vitek $^{\circledR} \mathbf{2}$ & Macro Etest $^{\circledR}$ & & & \\
\hline Case I & $\leq 1$ & 8 & 12 & 4 & hVISA (I.16) \\
\hline Case 2 & 2 & 8 & Not done & 4 & hVISA (I.00) \\
\hline Case 3 & 2 & 4 & 16 & 2 & hVISA (0.95) \\
\hline
\end{tabular}

Abbreviations: AUC, area under the curve; PAP, population analysis profile; hVISA, heteroresistant vancomycin-intermediate Staphylococcus aureus. 
between the two stages. Additionally, she received 3 months of oral fusidic acid and minocycline (based on sensitivities) after completion of the intravenous vancomycin.

Clinical and biochemical resolution of infection was initially demonstrated, but, 9 months later, there was a relapse of pain in the prosthetic hip with raised inflammatory markers. After consultation with the patient, a decision was made to explant the prosthesis and perform Girdlestone's procedure in the hope of eradicating the infection. This was accompanied by 4 weeks of vancomycin, followed by 2 weeks of linezolid with clinical improvement. Over the next 2 years, the patient had four relapses, based on the recurrence of symptoms and the growth of MRSA from surgical washouts of the hip pseudoarthrosis. During this time, she received vancomycin for a cumulative duration of 24 weeks, with a temporary clinical and biochemical resolution during each episode.

The last relapse prompted extensive debridement of the acetabulum, with operative samples sent for macro Etest, with a population analysis profile confirming hVISA (Table 1). Thus, pristinamycin was commenced and was continued as a suppressive therapy for 12 months. Over the subsequent 4 years, the patient remained well while off suppressive antibiotics. However, after sustaining a fall, she again developed pain in the region of the previously infected hip and elevated inflammatory markers. A periacetabular collection on magnetic resonance imaging led to concerns of recurrent infection. Vancomycin was commenced empirically and a subsequent surgical washout was performed. Operative cultures were negative, but pain and inflammatory markers started to resolve on vancomycin. Based on this, it was proposed as a relapse of MRSA infection. Due to the history of hVISA, pristinamycin was recommenced and continued indefinitely in tablet form. This was felt to be the most prudent approach, given the patient's 10-year history with a relapsing joint infection, even after total joint explantation. The patient is currently on suppressive antibiotic therapy, with no symptoms and controlled inflammatory markers.

\section{Case 3}

An 82-year-old man underwent an elective left total-knee arthroplasty. He had a complex cardiac history requiring warfarinization. On preadmission screening, he was noted to be MRSA positive. He had probably acquired MRSA during treatment for heart failure at another tertiary center. No vancomycin use was noted during that admission. The elective arthroplasty was uncomplicated and he received Vancomycin as antimicrobial prophylaxis preoperatively. He was discharged on day 11 , after an unremarkable postoperative course.
The patient re-presented the next day, complaining of excessive bleeding from his wound. Examination revealed superficial dehiscence, but, over the next week, there was continued deeper dehiscence. Negative-pressure wound therapy was applied based on plastic-surgical opinion. On day 18, a superficial wound swab revealed polymicrobial growth of methicillin-sensitive Staphylococcus aureus (MSSA), MRSA, and Klebsiella pneumonia. Although he remained apyrexial and inflammatory markers were not raised, a dual antibiotic therapy of vancomycin and ceftriaxone was commenced.

Despite these measures, the wound continued to deteriorate and he returned to the theater for irrigation and debridement on day 22. Operative findings revealed a large congealed hematoma, which was evacuated. Microbiological samples again revealed polymicrobial growth (MSSA, Pseudomonas aeruginosa, K. pneumoniae, and Bacteroides fragilis). However, as there was no evidence of MRSA on any operative sample, ticarcillin-clavulanate was commenced on day 26.

Following three further washouts, the wound was judged sterile, and gastrocnemius flaps and a split skin graft covered the tissue defect with plastic surgery. However, the wound began to dehisce superiorly at the margin of the flap and split skin graft, with prosthesis on show. After extensive discussion with the patient, it was decided to proceed with knee fusion on day 60. Operative cultures revealed a pure growth of MRSA, sensitive to vancomycin, on routine susceptibility testing (Vitek 2).

The patient was recommenced on vancomycin. However, due to the persistent nature of his infection, macro Etest and population analysis profiling was performed, which confirmed hVISA (Table 1). In anticipation of daptomycin use, sensitivities were performed and found to be daptomycin resistant (Vitek 2) (Table 1). The patient was then commenced on linezolid for 4 weeks and subsequently placed on a long-term dual therapy of oral pristinamycin and ciprofloxacin, with clinical resolution.

\section{Discussion \\ Clinical features}

These three cases present significant commonalities and differences (refer Table 2). The cases differ in their presence of predictors for hVISA (colonization with MRSA, prior vancomycin use, vancomycin treatment failure, and the presence of high-inoculum infections). ${ }^{3}$ Only Case 3 was colonized with MRSA prior to arthroplasty. Cases 1 and 2 acquired MRSA postoperatively, despite appropriately short 
Table 2 Clinical and surgical features

\begin{tabular}{lll}
\hline Clinical features & Case I & Case 2 \\
\hline MRSA colonized $^{\mathrm{a}}$ & $\mathrm{No}$ & Yes \\
Cumulative duration of vancomycin (weeks) $^{\mathrm{b}}$ & 0 & 24 \\
Number of failed two-stage revisions & $\mathrm{I}$ & 3 \\
Type of surgeryc & Above-knee amputation & Girdlestone's procedure \\
Outcome & Possible cure & Relapse necessitating indefinite \\
Alternative antibiotic & (no relapse after 6 years) & antibiotic suppression \\
\hline
\end{tabular}

Note: aPrior to initial arthroplasty; ${ }^{b}$ prior to detection of heteroresistant vancomycin-intermediate Staphylococcus aureus; ${ }^{\mathrm{c}}$ most recent surgery. Abbreviation: MRSA, methicillin-resistant Staphylococcus aureus.

courses of perioperative antibiotic prophylaxis. Only Case 1 was associated with vancomycin-treatment failure and prolonged use of vancomycin. Case 3 had a short course of vancomycin (7 days) when hVISA was detected, while Case 2 had no prior vancomycin exposure at all. This suggests that the mode of acquisition for hVISA can vary, implicating both glycopeptide selection pressure and nosocomial transmission.

There are plenty of common features. From an orthopedic point of view, there were appropriately early and aggressive "de-bulking" procedures, reflecting a high standard of care. There were four two-stage revisions among the three patients and in all instances they were performed early $(<1$ month from onset/relapse of symptoms). Girdlestone's procedure and amputation were performed as a last resort for cure of infection and, despite the former, there was microbiologically proven relapse. The only instance of knee fusion was partly due to patient preference and this warranted long-term suppressive antibiotic therapy. Given the proficient orthopedic interventions, the failure of treatment is largely suggestive of microbial factors. This leads us to discuss the microbiology of hVISA.

\section{Microbiology}

Low-level vancomycin resistance can arise in any strain of $S$. aureus, but almost all cases of hVISA reported to date have occurred in isolates of MRSA and were found predominantly in nosocomial settings. ${ }^{3}$ The main characteristics of clinically derived hVISA strains are a thickened cell wall, with reduced peptidoglycan cross-linking, and a reduced growth rate. It is thought that reduced cross-linking in the cell wall leads to greater numbers of "free" peptidoglycan side-chains, which serve as "decoy" binding sites for vancomycin. ${ }^{5}$ Thus, the vancomycin is unable to reach its site of activity at the cell membrane when it is incarcerated in the outer layer of the thickened cell wall.
The literature reveals that hVISA strains may demonstrate either stable or unstable heterogeneous resistance. ${ }^{6}$ This is supported by our observation that Cases 1 and 2 either had little or no prior exposure to vancomycin, possibly reflecting a stable phenotype. However, it is speculated that Case 3 demonstrates an acute progression of MRSA infection, which initially responded to vancomycin, correlating with an unstable phenotype.

A feature of PJIs is the presence of a high bacterial load. In vitro studies have shown that the presence of hVISA and a high bacterial load affect vancomycin activity and this may increase the risk of treatment failure. ${ }^{7}$ This was found to be the case even with vancomycin levels thought to be therapeutic in clinical settings. ${ }^{8,9}$

By definition, all three cases had heterogeneous populations of $S$. aureus, but Case 3 also had MSSA isolated from deep specimens. MSSA was cultured while the patient was on vancomycin, whereas the known colonizer, MRSA, was not found at that time. This finding may be attributed to the fact that vancomycin has been shown to be inferior to $\beta$-lactams when it comes to treating MSSA infections. These observations emphasize that antibiotic choice is crucial in influencing bacterial phenotype.

\section{Antibiotic choice}

Choice of antibiotic is a challenging issue, as demonstrated in other published studies where alternative antibiotics were of limited utility due to serious adverse effects, development of resistance, and futility. ${ }^{10-12}$ In our series, vancomycin use was optimized in all cases, as evidenced by the trough levels. This failed to bring about a clinical response, perhaps due to the nature of the PJIs and microbiological factors already mentioned. Linezolid was trialed and discontinued in Cases 2 and 3 for intractable nausea and significant myelosuppression, respectively. Case 1 tolerated a short course of linezolid and most probably achieved a cure after source control via amputation. Pristinamycin was well 
tolerated in Cases 2 and 3, and it has proven effective in suppressing infection.

Daptomycin was not an option for Cases 1 and 2, as they were diagnosed in 2005, prior to the availability of daptomycin in Australia. Case 3 was more recent, but the isolate demonstrated in vitro nonsusceptibility (Table 1), which precluded the use of daptomycin. The detection of daptomycin nonsusceptibility led us to examine the correlation between reduced daptomycin susceptibility and vancomycin resistance in $S$. aureus. Thus, for this case series, isolates from Cases 1 and 2 were also tested and found to be nonsusceptible to daptomycin.

\section{Daptomycin nonsusceptibility in hVISA}

A positive correlation between reduced daptomycin susceptibility and vancomycin resistance in hVISA has been proposed, with suggestions of a similar mechanism (a thickened cell wall), although this has not been confirmed. ${ }^{13}$ The SENTRY surveillance program reported that only 1 of $55(2 \%)$ hVISA strains had a daptomycin minimum inhibitory concentration $2 \mathrm{mg} / \mathrm{mL}$ or above. ${ }^{14}$ Thus, our observation that all three isolates exhibited daptomycin-glycopeptide cross-resistance was unexpected. A more recent study showed the highest rate, where 15 of $27(15 \%)$ hVISA strains demonstrated reduced susceptibility to daptomycin. ${ }^{15}$ The clinical significance of this microbiological observation is not known, as the bactericidal activity of daptomycin was maintained against hVISA strains in vitro. ${ }^{16,17}$ However, in clinical scenarios, caution is warranted, as failures of daptomycin therapy in hVISA infections have been documented. ${ }^{11,12,18-21}$ Interestingly, a recent paper reported seven cases of hVISA bacteremia (with daptomycin nonsusceptibility), where eradication was achieved with a combination of antistaphylococcal $\beta$-Lactams (ASBL) and daptomycin. ${ }^{22}$ The enhanced binding and activity of the daptomycin was attributed to the ASBL-mediated reduction in the surface charge of the cell wall.

\section{Conclusion}

This series highlights the complexities associated with the diagnosis and management of hVISA PJIs. Despite appropriate and timely surgical and medical intervention, unsatisfactory outcomes were achieved in all three cases, which was probably due to microbiological factors. hVISA demonstrates a high affinity for bone and prosthetic material, as well as the ability to resist antibiotic classes via its thickened cell wall, rendering eradication extremely difficult.

A review of the literature identifies various principles that should be considered for the successful management of future infections. A high index of suspicion for hVISA must be present in any MRSA bone, joint, or prosthetic infection, especially upon relapse or treatment failure. Routine screening for hVISA from all MRSA isolates is prudent. Early surgical source control and appropriate antibiotic therapy is crucial. Various antibiotic strategies have been suggested, such as using a non-cell wall acting agent (eg, linezolid) for high bacterial-load infections other than endocarditis. ${ }^{6}$ Combination therapy for synergy or potentiation (ASBL-daptomycin) may prove useful. Further research is critical for establishing the best antibiotic strategy for what is expected to be a growing problem.

In terms of prevention, strategies for vancomycin pharmacokinetic and pharmacodynamic optimization are important. Loading doses of vancomycin must be given serious consideration, along with meticulous monitoring of serum vancomycin concentration. This will not only improve vancomycin efficacy, but also minimize the risk of developing vancomycin heteroresistance.

\section{Disclosure}

The authors declare no conflicts of interest in this work. All authors participated in the research and appropriate ethical consent was obtained.

\section{References}

1. Peel TN, Buising KL, Choong PF. Prosthetic joint infection: challenges of diagnosis and treatment. ANZ J Surg. 2011;81(1-2):32-39.

2. Turnidge JD, Nimmo GR, Francis G. Evolution of resistance in Staphylococcus aureus in Australian teaching hospitals. Australian Group on Antimicrobial Resistance (AGAR). Med J Aust. 1996;164(2): 68-71.

3. Howden BP. Recognition and management of infections caused by vancomycin-intermediate Staphylococcus aureus (VISA) and heterogenous VISA (hVISA). Intern Med J. 2005;35(2):S136-S140.

4. Fong RK, et al. Clinical features and treatment outcomes of vancomycin-intermediate Staphylococcus aureus (VISA) and heteroresistant vancomycin-intermediate Staphylococcus aureus (hVISA) in a tertiary care institution in Singapore. Eur J Clin Microbiol Infect Dis. 2009;28(8):983-987.

5. Nannini E, Murray BE, Arias CA. Resistance or decreased susceptibility to glycopeptides, daptomycin, and linezolid in methicillin-resistant Staphylococcus aureus. Curr Opin Pharmacol. 2010;10(5):516-521.

6. Rong SL, Leonard SN. Heterogeneous vancomycin resistance in Staphylococcus aureus: a review of epidemiology, diagnosis, and clinical significance. Ann Pharmacother. 2010;44(5):844-850.

7. Rose WE, Leonard SN, Rossi KL, Kaatz GW, Rybak MJ. Impact of inoculum size and heterogeneous vancomycin-intermediate Staphylococcus aureus (hVISA) on vancomycin activity and emergence of VISA in an in vitro pharmacodynamic model. Antimicrob Agents Chemother. 2009;53(2):805-807.

8. Maor Y, Hagin M, Belausov N, Keller N, Ben-David D, Rahav G. Clinical features of heteroresistant vancomycin-intermediate Staphylococcus aureus bacteremia versus those of methicillin-resistant $S$. aureus bacteremia. J Infect Dis. 2009;199(5):619-624. 
9. Charles PG, Ward PB, Johnson PD, Howden BP, Grayson ML. Clinical features associated with bacteremia due to heterogeneous vancomycin-intermediate Staphylococcus aureus. Clin Infect Dis. 2004;38(3):448-451.

10. Cheong JW, Harris P, Oman K, Norton R. Challenges in the microbiological diagnosis and management of hVISA infections. Pathology. 2011;43(4):357-361.

11. van Hal SJ, Paterson DL, Gosbell IB. Emergence of daptomycin resistance following vancomycin-unresponsive Staphylococcus aureus bacteraemia in a daptomycin-naïve patient - a review of the literature. Eur J Clin Microbiol Infect Dis. 2011;30(5):603-610.

12. Cunha BA, Pherez FM. Daptomycin resistance and treatment failure following vancomycin for methicillin-resistant Staphylococcus aureus (MRSA) mitral valve acute bacterial endocarditis (ABE). Eur J Clin Microbiol Infect Dis. 2009;28(7):831-833.

13. Cui L, Tominaga E, Neoh H, Hiramatsu K. Correlation between reduced daptomycin susceptibility and vancomycin resistance in vancomycinintermediate Staphylococcus aureus. Antimicrob Agents Chemother. 2006;50(3):1079-1082.

14. Loza E, Morosini MI, Pascual A, et al. Comparative in vitro activity of daptomycin against gram-positive microorganisms: SENTRY surveillance program, Spain (2002-2006). Enferm Infecc Microbiol Clin. 2008;26(8):489-494. Spanish.

15. Kelley PG, Gao W, Ward PB, Howden BP. Daptomycin non-susceptibility in vancomycin-intermediate Staphylococcus aureus (VISA) and heterogeneous-VISA (hVISA): implications for therapy after vancomycin treatment failure. J Antimicrob Chemother. 2011;66(5):1057-1060.

16. Akins RL, Rybak MJ. Bactericidal activities of two daptomycin regimens against clinical strains of glycopeptide intermediate-resistant Staphylococcus aureus, vancomycin-resistant Enterococcus faecium, and methicillin-resistant Staphylococcus aureus isolates in an in vitro pharmacodynamic model with simulated endocardial vegetations. Antimicrob Agents Chemother. 2001;45(2):454-459.
17. Marco F, García-de-la-Mària C, Amero Y, et al. Daptomycin is effective in treatment of experimental endocarditis due to methicillin-resistant and glycopeptide-intermediate Staphylococcus aureus. Antimicrob Agents Chemother. 2008;52(7):2538-2543.

18. Kirby A, Mohandas K, Broughton C, et al. In vivo development of heterogeneous glycopeptide-intermediate Staphylococcus aureus (hGISA), GISA and daptomycin resistance in a patient with meticillin-resistant S. aureus endocarditis. J Med Microbiol. 2009;58(3):376-380.

19. Twele L, Moyen E, Zhang K, Dalton B, Church D, Conly J. Methicillinresistant Staphylococcus aureus endocarditis and de novo development of daptomycin resistance during therapy. Can J Infect Dis Med Microbiol. 2010;21(2):89-93.

20. Skiest DJ. Treatment failure resulting from resistance of Staphylococcus aureus to daptomycin. J Clin Microbiol. 2006;44(2):655-656.

21. Hayden MK, Rezai K, Hayes RA, Lolans K, Quinn JP, Weinstein RA. Development of daptomycin resistance in vivo in methicillin-resistant Staphylococcus aureus. J Clin Microbiol. 2005;43(10):5285-5287.

22. Dhand A, et al. Use of antistaphylococcal \{beta\}-lactams to increase daptomycin activity in eradicating persistent bacteremia due to methicillin-resistant Staphylococcus aureus: role of enhanced daptomycin binding. Clin Infect Dis. 2011;53(2):158-163.

23. Clinical and Laboratories Standards Institute. Performance standards for Antimicrobial Susceptibility Testing: Eighteenth International Supplement M100-S18. Wayne, PA: Clinical and Laboratory Standards Institute; 2008.
Orthopedic Research and Reviews

\section{Publish your work in this journal}

Orthopedic Research and Reviews is an international, peer-reviewed, open access journal focusing on the patho-physiology of the musculoskeletal system, trauma, surgery and other corrective interventions to restore mobility and function. Advances in new technologies, materials, techniques and pharmacological agents are particularly welcome. The journal welcomes

\section{Dovepress}

original research, clinical studies, reviews \& evaluations, expert opinion and commentary, case reports and extended reports. The manuscript management system is completely online and includes a very quick and fair peer-review system, which is all easy to use. Visit http://www.dovepress. com/testimonials.php to read real quotes from published authors. 